reasons for their increased risk is of importance in designing effective interventions. We sought to identify characteristics that might explain re-infection among these men.

Methods Between 2004-2010 the LGV Enhanced Surveillance system collected individual-level cross-sectional data on confirmed LGV episodes. We compared the baseline characteristics of men with repeat LGV episodes to men with a single reported episode and we report univariate logistic regression or Fisher's exact test where appropriate. Results In the dataset 1215 individuals had a single reported infection and 62 had a known repeat infection. The mean age was 38 which did not differ between groups. Men with re-infection had higher prevalence of HIV than those with one episode $97 \%$ versus $79 \%, \mathrm{P}<0.001)$, concurrent hepatitis C (PCR) $(19 \%$ versus $9 \%$, OR $2.19,95 \%$ CI $1.05,4.59)$ and gonorrhoea infection $(29 \%$ versus $16 \%$, OR $2.16,95 \%$ CI $1.21,3.84)$. Repeaters were also more likely to be seen in a clinic in London (81\% versus $68 \%$, OR $2.00,95 \%$ CI 1.05 , $3.80)$ and they reported higher levels of unprotected sex including insertive and receptive unprotected sex. However, a high proportion of non-repeaters also reported unprotected sex and the difference was not statistically significant.

Conclusions LGV repeaters display characteristics traditionally attributed to core groups, but behavioural characteristics alone did not explain LGV re-infection among these patients. LGV repeaters have a high prevalence of STI co-infections which is of clinical and epidemiological relevance, and may suggest their position in the sexual network is contributing to the heightened risk for STI acquisition. Further research is needed to ascertain this.

\section{P3.142 EPIDEMIOLOGY OF STI IN MEN HAVING SEX WITH MEN IN ITALY, 1991-2010}

doi:10.1136/sextrans-2013-051184.0601

M Salfa, V Regine, L Camoni, M Raimondo, B Suligoi, the Italian STI Surveillance Working Group. Istituto Superiore di Sanità, Rome, Italy

Background In recent years, sexually transmitted infections (STI) have been increasing among men who have sex with men (MSM) in several industrialised countries. The objective of this study was to assess socio-demographic, behavioural and clinical characteristics of MSM with STI, in Italy.

Methods Data were obtained from the Italian Sentinel STI Surveillance System based on a network of 12 specialised clinical centres located in large cities (1991-2010). All MSM are offered HIV testing and serostatus is recorded.

Results Between 1991 and 2010, 85,073 STI cases were reported; $13,081(15.6 \%)$ were diagnosed among MSM. The annual number of STI cases remained stable over time, whereas the proportion of MSM with STI increased from $11.2 \%$ in 1991 to $17.5 \%$ in 2010 . The most frequent diagnoses among MSM were: genital warts (33.3\%), primary and secondary syphilis (19.5\%), and gonoccocal urethritis $(15.4 \%)$. The annual number of cases of primary and secondary syphilis remained stable until 2000 (about 24 cases/year) and then increased ten-fold in 2005 (No. 325) compared to 2000, with a slight decrease after 2005. Overall, among the 10,850 (82.9\%) MSM with STI who underwent HIV testing, the HIV prevalence was $21.1 \%$, showing a decrease during the study period from $28.1 \%$ in 1991 to $21.3 \%$ in 2010. Among the 2,288 HIV-positive MSM, 35.7\% were new HIV diagnoses.

Conclusion The proportion of MSM with STI increased between 1991 and 2010. In 2010, compared to heterosexuals, HIV prevalence among MSM was eight times higher and the proportion of primary and secondary syphilis was six times higher.

These results stress the need for prevention campaigns targeted at reducing the spread of STI among MSM, and the need for an active proposal of HIV testing (including the opt-out approach) among STI patients.

\section{P3.143 THE EPIDEMIOLOGY OF SEXUALLY TRANSMITTED INFECTIONS AMONG MEN WHO HAVE SEX WITH MEN IN MAINLAND CHINA: A META-ANALYSIS AND DATA SYNTHESIS}

doi:10.1136/sextrans-2013-051184.0602

E P F Chow, Y Wang, D P Wilson, L Zhang. The Kirby Institute, University of New South UK, Darlinghurst, NSW, Australia

Background HIV and syphilis prevalence has increased substantially among men who have sex with men (MSM) in China. This study aims to assess the magnitude in other sexually transmitted infections (STIs) among this population.

Methods Chinese and English peer-reviewed articles were searched from five electronic databases (PubMed, Embase, Wanfang Data, VIP Chinese Journal Database and China National Knowledge Infrastructure) up to Dec 2012. Pooled prevalence estimate for each STI from available studies were calculated. Odds ratios for STIs prevalence among MSM were compared with the adults in the general population and male sex workers (MSW).

Results Eighty-eight articles (11 in English and 77 in Chinese) with a total of 35203 MSM were included in this review. The national prevalence level of STIs between 2003 and 2011 were: $6.32 \%(3.54-11.02 \%)$ for chlamydia, $1.86 \%(1.27-2.73 \%)$ for gonorrhoea, $8.92 \%$ (7.75-10.24\%) for HBV, $1.25 \%$ (1.00-1.55\%) for HCV, $66.25 \%(57.38-74.10 \%)$ for any HPV genotype, $10.64 \%(6.24-$ $17.57 \%$ ) for HSV-2, and $13.45 \%$ (11.78-15.23\%) for syphilis. MSM have consistently higher risk of STIs than the general Chinese population (chlamydia: $\mathrm{OR}=1.43,1.31-1.56$; gonorrhoea: $\mathrm{OR}=2.42$, 1.63-3.59; HBV: OR = 1.97, 1.57-2.48; HCV: OR = 48.40, 6.38367.09; syphilis: $\mathrm{OR}=28.38,28.38-31.14)$. However, as a subgroup of MSM, MSWs were 1.43 (1.31-1.56), 2.42 (1.63-3.59) and 2.26 (1.37-3.72) more likely to be infected with chlamydia, gonorrhoea and HCV than the broader MSM population.

Conclusion Prevalence levels of STIs among MSM in China are greater than levels in the general population and MSWs have greater prevalence of STIs compared with the broader MSM population.

\section{P3.144 STD CLINICS SERVE AN ECONOMICALLY DISADVANTAGED SUBSET OF THE WIDER POPULATION OF MEN WHO HAVE SEX WITH MEN (MSM) WITH BACTERIAL SEXUALLY TRANSMITTED INFECTIONS (STI)}

doi:10.1136/sextrans-2013-051184.0603

${ }^{1,2}$ R P Kerani, ${ }^{1,2} \mathrm{~J}$ Dombrowski, 'L Barbee, 1,2D A Katz, 1,2M R Golden. 'University of Washington, Seattle, WA, United States; ${ }^{2}$ Public Health - Seattle \& King County, Seattle, WA, United States

Background U.S. STD clinics are closing. MSM are a priority population for STD treatment. We evaluated differences between MSM diagnosed with STI in an STD clinic and MSM diagnosed in other venues.

Methods We randomly sampled and interviewed MSM reported with gonorrhoea, chlamydial infection, or early syphilis in King County, Washington, 2007-2012. We weighted for interview nonresponse based on race, ethnicity, STI diagnosis, STD clinic diagnosis (vs. elsewhere), and year.

Results A total of 2,865 (38\%) of 7,568 MSM cases were randomly selected, of whom 2107 (73.5\%) were interviewed; 1275 (61\%) interviewed men were STD clinic patients. STD clinic patients were less likely to have stable housing ( $91 \%$ vs. $95 \%, p=0.003$ ) or a college degree ( $34 \%$ vs. $44 \%, p<0.0001)$, and more likely to be unemployed ( $28 \%$ vs. $23 \%, p=0.01$ ). Both groups were similar in terms of age, race, ethnicity, and HIV status. STD clinic patients were more likely to be diagnosed with gonorrhoea (57\% vs. $47 \%$, $\mathrm{p}<0001$ ), to report a prior diagnosis of bacterial STI (68\% vs.55\% $\mathrm{p}<0.0001)$, and to have sought care because of symptoms (45\% vs. 